\title{
Gastrointestinal Tract Development in Unweaned Calves Feeding Different Amounts of Milk and Different Starters
}

\author{
Tian Zhang*, Zhiqiang Wu*, Qiuling Hou, Yun Wang, Zhiyong Hu, Xueyan Lin\#, Zhonghua Wang\# \\ College of Animal Science, Shandong Agricultural University, Taian, China \\ Email: "zhwang@sdau.edu.cn, "xueyanlin2005@163.com
}

How to cite this paper: Zhang, T., Wu, Z.Q., Hou, Q.L., Wang, Y., Hu, Z.Y., Lin, X.Y. and Wang, Z.H. (2018) Gastrointestinal Tract Development in Unweaned Calves Feeding Different Amounts of Milk and Different Starters. Advances in Bioscience and Biotechnology, 9, 289-310. https://doi.org/10.4236/abb.2018.97019

Received: May 8, 2018

Accepted: July 3, 2018

Published: July 6, 2018

Copyright $(9) 2018$ by authors and Scientific Research Publishing Inc. This work is licensed under the Creative Commons Attribution International License (CC BY 4.0).

http://creativecommons.org/licenses/by/4.0/

(c) $\underset{\mathrm{EY}}{\mathrm{i}}$ Open Access

\begin{abstract}
This study was conducted to determine the effect of different starter diets and different amounts of milk on growth performance and gastrointestinal tract development in unweaned calves. 16 calves were assigned to 4 groups, 4 calves in each group. These four groups received the following treatments respectively: 1) high milk (6 L) + low starch (21\%), high NDF (28\%), high molasses (10\%) starter (HMLS group); 2) high milk (6L) + high starch (40\%), low NDF (14\%), low molasses (5\%) starter (HMHS group); 3 ) low milk (3 L) + low starch (21\%), high NDF (28\%), high molasses (10\%) starter (LMLS group); 4) low milk (3 L) + high starch (40\%), low NDF (14\%), low molasses (5\%) starter (LMHS group). The trial was of $2 \times 2$ factorial design. All calves had free access to hay and water. Results showed that the low milk allowance increased calf concentrate dry matter intake (DMI) and total DMI, reduced body height at 4 weeks of age, reduced heart girth at 6 weeks of age $(P<0.05)$. The low milk allowance increased complex stomach full weight, reticulorumen full weight, and the percentages of stomach full weight and reticulorumen full weight over body weight $(P<0.05)$. The low starch, high fiber, high molasses starters reduced the complex stomach full weight, the proportion of the complex stomach empty weight over body weight, reticulorumen empty weight, abomasum full weight, the proportion of abomasum empty weight over body weight and reticulorumen volume $(P<0.05)$. The low milk allowance increased calf intestine length, small intestine full weight, the proportion of small intestine full weight over body weight, but reduced the jejunum villus width $(P<0.05)$, increased ruminal $\mathrm{pH}(P<0.05)$. The low milk allowance reduced papilla length in rumen anterior ventral blind sac, but increased papilla length in the posterior ventral sac $(P<0.05)$.
\end{abstract}




\section{Keywords}

Calf, Starter Kinds, Milk Amount, Gastrointestinal Development, Growth Performance

\section{Introduction}

Traditional calf starters are rich in readily fermentable starch. Upon ruminal microbial fermentation, starters favor short-chain volatile fatty acid (VFA) production, which stimulates rumen development in unweaned calves [1]. However, high-starch diets can cause rumen $\mathrm{pH}$ decrease [2], resulting in subacute ruminal acidosis [3]. Although digestible fiber in starters may increase rumen $\mathrm{pH}$, preventing subacute ruminal acidosis, the fermentation of fiber favors the production of acetic acid, weakening the stimulating effect on epithelium growth, because molasses contain a large portion of sucrose, which favors butyric acid production. Studies have shown that high molasses in starters are more conducive to promoting rumen development [4]. Therefore, we hypothesize that partial replacement of starch with fiber and molasses together may alleviate subacute ruminal acidosis without weakening calf rumen development.

Solid feed produces volatile fatty acids by rumen microbial fermentation, which promotes rumen epithelium development in unweaned calves, while the physical form of the solid feed also affects the development of rumen volume and muscle. Milk is not conducive to the development of rumen and reticulum in calves. Although the complex stomach will grow, papilla development will be inhibited and the ruminal wall will be thinner. Therefore, it has been considered a good way to limit the amount of milk, which could increase solid feed intake, thereby contributing to the development of rumen epithelium [5]. However, few studies have been conducted on this issue. Kristensen et al. reported that, when calves were fed more milk in the diet, the rumen may still have a lower $\mathrm{pH}$ and higher concentration of VFA. Rumen epithelial papilla length was not affected by the added amount of milk [6] [7]. Therefore, whether the amount of milk affects calf rumen epithelial development is inconclusive so far.

Therefore, this study aims to investigate how traditional high starch starters and low starch high fiber high molasses starters affect the development of the gastrointestinal tract in calves, and how different amounts of milk in diet affect gastrointestinal development, providing a references for the development of gastrointestinal tract of calves.

\section{Materials and Methods}

\subsection{Materials and Design}

16 healthy newborn Holstein dairy bull calves, weighing about $40 \mathrm{~kg}$, were randomly assigned to four groups, each group with 4 calves. The test was a $2 \times 2$ factorial design. The groups were respectively feed 1) high milk (6 L) + low 
starch (21\%), high NDF (28\%), high molasses (10\%) starter (HMLS group); 2) high milk (6 L) + high starch (40\%), low NDF (14\%), low molasses (5\%) starter (HMHS group); 3) low milk (3 L) + low starch (21\%), high NDF (28\%), high molasses (10\%) starter (LMLS group); 4) Low milk (3 L) + high starch (40\%), low NDF (14\%), low molasses (5\%) starter (LMHS group). All calves were allowed free access to water, starter diets, and chopped oat hay. Two weeks before the trial, all calves were fed $6 \mathrm{~L}$ of milk. From the second 2 week on, milk fed to calves in groups 3 and 4 was reduced to $3 \mathrm{~L}$. Beginning from 1 week old, calves were induced to starters. Calves were weaned at 60 days of age. Three healthy calves from each group were slaughtered for measurement at 60 days of age.

Diets

Milk used in this trial contained $3.4 \%$ to $3.8 \%$ of fat, $3.0 \%$ to $3.5 \%$ of protein. The ingredients and nutritional values of starter diets were shown in Table 1 and Table 2.

\subsection{Sample Collection}

1) The collection and processing of blood

Table 1. Composition of different starters.

\begin{tabular}{ccc}
\hline & High starch starter & Low starch starter \\
\hline Corn (\%) & 58.03 & 28.36 \\
Soy bean (\%) & 22.50 & 21.83 \\
Bran (\%) & 9.77 & 9.87 \\
Soybean skin (\%) & 0.00 & 25.24 \\
Molasses (\%) & 5.00 & 10.00 \\
Stone powder (\%) & 1.50 & 1.50 \\
Salt (\%) & 0.40 & 0.40 \\
CaHPO4 (\%) & 0.80 & 0.80 \\
Premix (\%) & 2.00 & 2.00 \\
\hline
\end{tabular}

Note: Per kg of premix can meet all microelements and vitamins need for calves.

Table 2. Nutrient contents of different starters and oat grass (as\% of DM).

\begin{tabular}{cccc}
\hline Ingredient & High starch starter & Low starch starter & Oat grass \\
\hline DM (\%) & 88.06 & 88.93 & 90.32 \\
ME (M)/kg) & 12.44 & 11.34 & 7.91 \\
CP (\%) & 18.66 & 18.66 & 5.82 \\
Starch (\%) & 40.63 & 21.92 & 7.31 \\
NDF (\%) & 14.1 & 28.74 & 61.17 \\
Ash (\%) & 9.05 & 9.18 & 6.79 \\
Ca (\%) & 1.10 & 1.07 & 0.17 \\
P (\%) & 0.55 & 0.55 & 0.37 \\
\hline
\end{tabular}

Note: The compositions of starters were calculated from ingredients, and oat compositions were measured. 
At the end of the trial, three hours after the morning feeding, blood samples were collected from the Jugular vein, $30 \mathrm{ml}$ from each calf. Blood samples were injected into $10 \mathrm{ml}$ centrifuge tubes and allowed to stand at room temperature for about $30 \mathrm{~min}$. When serum emerged, blood samples were spun at $3000 \mathrm{rpm}$ for $10 \mathrm{~min}$. Serum was dispensed into $1.5 \mathrm{ml}$ centrifuge tubes, stored at $-20^{\circ} \mathrm{C}$ until test.

2) Collection and measurement of rumen fluid samples

Reticulorumen was opened and the chime was collected, filtered with three layers of gauze, then immediately measured with a $\mathrm{pH}$ meter, dispensed into four $50 \mathrm{ml}$ centrifuge tubes, $50 \mathrm{ml}$ each tube, and immediately stored at $-20^{\circ} \mathrm{C}$ for determination of volatile fatty acid concentrations.

3) Gastrointestinal tissue samples

Three tissue samples were collected from each of the anterior dorsal sac, posterior dorsal sac, anterior ventral sac, and posterior ventral sac of the rumen. Each tissue sample was $1 \mathrm{~cm} \times 1 \mathrm{~cm}$ in size. Samples were cleaned with saline immediately then fixed in $10 \%$ neutral formalin solution. Three samples of duodenal tissue, each $1 \mathrm{~cm}$ long, were collected from a region of about $20 \mathrm{~cm}$ away from pylorus, rinsed with saline, immediately fixed with $10 \%$ neutral formalin. Three pieces of $1 \mathrm{~cm}$ long jejunum tissue samples were removed from the midsection of the jejunum, rinsed with saline, immediately fixed in $10 \%$ neutral formalin solution. Three ilium samples were collected from a section of about 20 $\mathrm{cm}$ away from the ileocecal mouth, rinsed with saline, immediately fixed in $10 \%$ neutral formalin solution.

\subsection{Determination Methods}

1) Determination of feed ingredients and nutrients of oat hay

Dry matter (DM) was measured according to "Feed Analysis and Feed Quality Testing Technologies" (edited by Liying Zhang). The crude protein (CP) was determined using a Kjeldahl analyzer. The total energy (GE) was measured using a semi-automatic oxygen bomb calorimeter (PARR 6200, USA). Starch was determined using the perchloric acid hydrolysis-anthracene colorimetry method. Neutral detergent fiber (NDF) was determined with the Van Soest method. Ash, Calcium (Ca), and Phosphorus (P) were measured according "Feed Analysis and Feed Quality Testing Technologies" (edited by Liying Zhang). The milk protein and milk fat were measured using a milk ingredient analyzer (Foss 78110, USA).

2) Quantitation of starters and oat hay intake

Starting from 7 days of age, certain amount of the starter and oat hay were presented to calves, twice a day. The next day before feeding, the residual quantity was recorded, and the daily dry matter intake (DMI) was calculated.

3) Measurements of body weight, body size and empty body weight

Body weight, body height, body length, heart girth, cannon circumference were measured at $0,2,4,6,8$ weeks and 60 days of age before milk feeding in the morning. The entire measurement process was completed by the same person. The empty weight was the final body weight of 60 -day-old calves minus ga- 
strointestinal chyme weight.

4) Determination of stomach Indexes

Isolated reticulorumen, omasum, abomasum (with chyme) were respectively weighed with electronic scales, rinsed with water, then weighed again (empty weight). After closing the opening of one end with a thin string, the reticulorumen was set flat on the ground then filled with water until overflow. The amount of water in the reticulorumen was measured, was considered the rumen volume. The omasum volume was measured the same way.

5) Determination of intestinal Indexes

Isolated small intestine, large intestine (with chyme) were weighed respectively with electronic scales, washed with water, then weighed again using electronic scales to obtain empty weight. Organ weight percentages were calculated by dividing the organ weight by the empty body weight. The lengths of intestinal segments were measured with a tape measure after removal of mesentery and straightened on the ground.

6) Serum parameters and VFA

Concentrations of serum glucose, total protein and urea were measured using an automatic biochemical analyzer (Hitachi 7020). The concentration of insulin and BHBA was determined using enzyme-linked immunosorbent assay kits (Shanghai BPRO). The IGF 1 concentration was determined in Taian Central Hospital. VFA concentrations in rumen fluid were measured using a gas chromatograph system (Shimadzu, GC-17A).

7) Rumen papillae and small intestine villi

Tissue sections were used to measure the rumen papillae length, width and muscular wall thickness, small intestine villi segment length, width and muscle thickness. Three different points were selected at random, and at each point, 10 good rumen papillae were selected at random to measure the length, width, muscle thickness. Intestinal villi were measured the same way.

\subsection{Data Analysis}

Data were analyzed using Factorial ANOVA in SAS 8.0, with the model of effects up to 2-way interactions. Duncan's multiple test were used for multiple comparisons of means. Differences were considered very significant if $P<0.01$, and significant if $0.01<P<0.05$, a trend if $0.05<P<0.1$. Means were not considered difference if $P>0.1$.

\section{Results}

\subsection{Dry Matter Intake and Body Weight}

As seen from Table 3, the low milk allowance increased concentrate DM intake (DMI) and total DMI $(P<0.05)$, however, milk allowance had no effect on the final weight and average daily gain (ADG, $P>0.1$ ). Different starters had no effect on calf DMI, final body weight and ADG $(P>0.1)$. There were interactions between the concentrate DMI and hay DMI, and between milk allowance and 
Table 3. Effect of different treatments on DMI and ADG of calves.

\begin{tabular}{cccccccccc}
\hline & \multicolumn{3}{c}{ Treatment group } & & \multicolumn{3}{c}{$P$} \\
\cline { 2 - 3 } & HMLS & HMHS & LMLS & LMHS & & SEM & & F & M $\times$ F \\
\hline $\begin{array}{c}\text { Concentrate DMI } \\
\text { (g/d) }\end{array}$ & 345.11 & 284.86 & 386.62 & 496.78 & 26.24 & 0.04 & 0.65 & 0.14 \\
Hay DMI (g/d) & 71.62 & 105.37 & 133.90 & 98.70 & 7.23 & 0.09 & 0.96 & 0.04 \\
$\begin{array}{c}\text { Total DMI (g/d) } \\
\text { Weight at birth }\end{array}$ & 416.73 & 390.23 & 520.52 & 595.48 & 28.01 & 0.02 & 0.68 & 0.39 \\
$\quad$ (kg) & 40.77 & 45.03 & 43.78 & 43.56 & 0.77 & 0.63 & 0.22 & 0.18 \\
Final weight (kg) & 79.00 & 80.05 & 77.13 & 78.46 & 1.06 & 0.44 & 0.59 & 0.95 \\
ADG (kg/d) & 0.64 & 0.58 & 0.56 & 0.58 & 0.02 & 0.32 & 0.70 & 0.34 \\
\hline
\end{tabular}

Note: $\mathrm{M}$ represents the effect of milk allowance. $\mathrm{F}$ represents the effect of starter type and $\mathrm{M} \times \mathrm{F}$ represents interactions between milk allowance and the starters. HMLS group : high milk $(6 \mathrm{~L})+$ low starch $(21 \%)$, high NDF (28\%), high molasses (10\%) starter; HMHS group: high milk (6 L) + high starch (40\%), low NDF (14\%), low molasses (5\%) starter; LMLS group: low milk (3 L) + low starch (21\%), high NDF (28\%), high molasses (10\%) starter LMHS group: low milk (3 L) + high starch (40\%), low NDF (14\%), low molasses (5\%) starter.

hay DMI $(P<0.05)$. Calves of HMHS and LMLS had the largest hay DMI $(P<$ $0.05)$.

\subsection{Body Height, Body Length}

As seen from Table 4, the high milk allowance increased the body height of calves $(P<0.05)$ at 4 weeks of age, but did not affect the height at 2, 6, 8 weeks and 60 days of age. The high milk allowance did not influence $(P>0.1)$ the daily variation of height throughout the course of the experiment. The high milk allowance showed a tendency of increasing daily changes of body length $(0.05<P$ $<0.01)$. The high starch low-fiber low molasses starter tended to increase the body height at 6 weeks of age $(0.05<P<0.01)$. Different starters did not significantly affect the body length during the entire trial period $(P>0.1)$.

\subsection{Effect of Dietary Treatments on Heart Girth and Cannon Circumference}

As seen in Table 5, the high milk allowance increased the heart girth at 6 weeks of age $(P<0.05)$, had no effect on the heart girth at 2, 4, 8 weeks and 60 days of age $(P>0.1)$. Milk allowance showed more significant impact on the cannon circumference. The high milk allowance increased the cannon circumference at 4,8 weeks of age $(P<0.05)$, increased the canon circumference at 6 weeks and 60 days of age $(P<0.01)$, but had no effect on daily changes of cannon circumference $(P>0.1)$. Starters had no effect on the heart girth and cannon circumference at 2, 4, 6, 8 weeks and 60 days of age, and no influence on daily changes of heart girth and cannon circumference $(P>0.1)$.

\subsection{Effect of Different Dietary Treatments on Daily Gain of Calves Each Week-Old Stage}

Table 6 shows that the high milk allowance increased daily weight gain during 
Table 4. Effect of different dietary treatments on body height and body length of calves.

\begin{tabular}{|c|c|c|c|c|c|c|c|c|c|}
\hline & \multirow{2}{*}{ Age } & \multicolumn{4}{|c|}{ Treatment } & \multirow{2}{*}{ SEM } & \multicolumn{3}{|c|}{$P$} \\
\hline & & HMLS & HMHS & LMLS & LMHS & & M & F & $\mathrm{M} \times \mathrm{F}$ \\
\hline \multirow{7}{*}{$\begin{array}{l}\text { Body } \\
\text { height }\end{array}$} & Birth $(\mathrm{cm})$ & 76.40 & 77.17 & 75.60 & 75.57 & 0.34 & 0.12 & 0.61 & 0.58 \\
\hline & $2 \mathrm{w}(\mathrm{cm})$ & 77.40 & 78.70 & 76.40 & 76.83 & 0.47 & 0.16 & 0.38 & 0.66 \\
\hline & $4 \mathrm{w}(\mathrm{cm})$ & 79.37 & 83.03 & 78.73 & 78.27 & 0.58 & 0.05 & 0.21 & 0.11 \\
\hline & $6 \mathrm{w}(\mathrm{cm})$ & 84.27 & 87.53 & 84.13 & 86.20 & 0.69 & 0.61 & 0.09 & 0.67 \\
\hline & $8 \mathrm{w}(\mathrm{cm})$ & 89.43 & 90.70 & 88.60 & 89.70 & 0.69 & 0.53 & 0.42 & 0.95 \\
\hline & $60 \mathrm{~d}(\mathrm{~cm})$ & 90.43 & 91.47 & 89.40 & 90.63 & 0.56 & 0.46 & 0.37 & 0.94 \\
\hline & $\begin{array}{l}\text { Daily change } \\
\qquad(\mathrm{cm} / \mathrm{d})\end{array}$ & 0.23 & 0.24 & 0.23 & 0.25 & 0.01 & 0.81 & 0.50 & 0.75 \\
\hline \multirow{7}{*}{$\begin{array}{l}\text { Body } \\
\text { length }\end{array}$} & Birth $(\mathrm{cm})$ & 66.67 & 67.80 & 68.37 & 69.70 & 0.77 & 0.28 & 0.45 & 0.95 \\
\hline & $2 \mathrm{wk}(\mathrm{cm})$ & 72.23 & 74.87 & 74.73 & 74.73 & 0.82 & 0.49 & 0.44 & 0.44 \\
\hline & $4 \mathrm{wk}(\mathrm{cm})$ & 76.00 & 79.13 & 76.93 & 77.53 & 0.53 & 0.76 & 0.12 & 0.27 \\
\hline & $6 \mathrm{wk}(\mathrm{cm})$ & 80.33 & 82.03 & 82.30 & 80.90 & 0.60 & 0.74 & 0.90 & 0.23 \\
\hline & $8 \mathrm{wk}(\mathrm{cm})$ & 88.13 & 87.87 & 86.20 & 87.37 & 0.86 & 0.50 & 0.80 & 0.69 \\
\hline & $60 \mathrm{~d}(\mathrm{~cm})$ & 89.03 & 89.23 & 86.97 & 89.23 & 0.60 & 0.43 & 0.35 & 0.43 \\
\hline & $\begin{array}{l}\text { Daily change } \\
(\mathrm{cm} / \mathrm{d})\end{array}$ & 0.37 & 0.36 & 0.31 & 0.33 & 0.01 & 0.08 & 0.99 & 0.53 \\
\hline
\end{tabular}

Note: $\mathrm{M}$ denotes the effect of milk allowance, $\mathrm{F}$ denotes the effect of starters, and $\mathrm{M} \times \mathrm{F}$ denotes the interaction between the milk allowance and starters. HMLS group: high milk (6 L) + low starch (21\%), high NDF (28\%), high molasses (10\%) starter; HMHS group: high milk (6 L) + high starch (40\%), low NDF (14\%), low molasses (5\%) starter; LMLS group: low milk (3 L) + low starch (21\%), high NDF (28\%), high molasses (10\%) starter LMHS group: low milk (3 L) + high starch (40\%), low NDF (14\%), low molasses (5\%) starter.

Table 5. Effect of different treatments on heart girth and cannon circumference.

\begin{tabular}{|c|c|c|c|c|c|c|c|c|c|}
\hline & \multirow{2}{*}{ Age } & \multicolumn{4}{|c|}{ Treatment } & \multirow{2}{*}{ SEM } & \multicolumn{3}{|c|}{$P$} \\
\hline & & HMLS & HMHS & LMLS & LMHS & & M & $\mathrm{F}$ & $\mathrm{M} \times \mathrm{F}$ \\
\hline \multirow{7}{*}{ Heart girth } & Birth $(\mathrm{cm})$ & 77.50 & 80.83 & 79.63 & 78.17 & 0.81 & 0.87 & 0.58 & 0.18 \\
\hline & $2 \mathrm{wk}(\mathrm{cm})$ & 82.43 & 84.00 & 83.03 & 81.90 & 0.96 & 0.71 & 0.91 & 0.50 \\
\hline & $4 \mathrm{wk}(\mathrm{cm})$ & 86.30 & 86.53 & 87.00 & 86.63 & 0.90 & 0.83 & 0.97 & 0.87 \\
\hline & $6 \mathrm{wk}(\mathrm{cm})$ & 92.30 & 92.23 & 91.13 & 88.20 & 0.55 & 0.05 & 0.21 & 0.23 \\
\hline & $8 \mathrm{wk}(\mathrm{cm})$ & 97.00 & 96.67 & 96.93 & 95.43 & 0.56 & 0.58 & 0.44 & 0.62 \\
\hline & $60 \mathrm{~d}(\mathrm{~cm})$ & 98.23 & 98.10 & 98.83 & 98.13 & 0.54 & 0.80 & 0.75 & 0.82 \\
\hline & $\begin{array}{l}\text { Daily change } \\
\qquad(\mathrm{cm} / \mathrm{d})\end{array}$ & 0.35 & 0.29 & 0.32 & 0.33 & 0.01 & 0.86 & 0.25 & 0.09 \\
\hline \multirow{7}{*}{$\begin{array}{c}\text { Cannon } \\
\text { circumference }\end{array}$} & Birth $(\mathrm{cm})$ & 11.70 & 11.83 & 11.47 & 11.47 & 0.13 & 0.26 & 0.80 & 0.80 \\
\hline & $2 \mathrm{wk}(\mathrm{cm})$ & 11.87 & 12.10 & 11.70 & 11.57 & 0.08 & 0.07 & 0.77 & 0.30 \\
\hline & $4 \mathrm{wk}(\mathrm{cm})$ & 12.13 & 12.17 & 11.80 & 11.60 & 0.08 & 0.02 & 0.61 & 0.48 \\
\hline & $6 \mathrm{wk}(\mathrm{cm})$ & 12.40 & 12.27 & 12.00 & 11.77 & 0.07 & 0.01 & 0.20 & 0.71 \\
\hline & $8 \mathrm{wk}(\mathrm{cm})$ & 12.93 & 12.63 & 12.27 & 12.50 & 0.06 & 0.01 & 0.80 & 0.07 \\
\hline & $60 \mathrm{~d}(\mathrm{~cm})$ & 12.97 & 12.77 & 12.40 & 12.50 & 0.08 & 0.01 & 0.68 & 0.24 \\
\hline & $\begin{array}{l}\text { Daily change } \\
(\mathrm{cm} / \mathrm{d})\end{array}$ & 0.020 & 0.015 & 0.016 & 0.017 & 0.01 & 0.66 & 0.64 & 0.32 \\
\hline
\end{tabular}

Note: $\mathrm{M}$ denotes the effect of milk allowance. $\mathrm{F}$ denotes the effect of starters and $\mathrm{M} \times \mathrm{F}$ denotes the interaction between milk allowance and starters. HMLS group: high milk (6 L) + low starch (21\%), high NDF (28\%), high molasses (10\%) starter; HMHS group: high milk (6 L) + high starch (40\%), low NDF (14\%), low molasses (5\%) starter; LMLS group: low milk (3 L) + low starch (21\%), high NDF (28\%), high molasses (10\%) starter LMHS group: low milk (3 L) + high starch (40\%), low NDF (14\%), low molasses (5\%) starter. 
Table 6. Effect of different treatments on ADG during different weeks of age.

\begin{tabular}{|c|c|c|c|c|c|c|c|c|}
\hline & \multicolumn{4}{|c|}{ Treatment } & \multirow{2}{*}{ SEM } & \multicolumn{3}{|c|}{$P$} \\
\hline & HMLS & HMHS & LMLS & LMHS & & M & $\mathrm{F}$ & $\mathrm{M} \times \mathrm{F}$ \\
\hline $\begin{array}{c}0 \text { - } 2 \text { week ADG } \\
(\mathrm{kg} / \mathrm{d})\end{array}$ & 0.40 & 0.31 & 0.34 & 0.41 & 0.03 & 0.80 & 0.89 & 0.32 \\
\hline $\begin{array}{c}2-4 \text { week ADG } \\
(\mathrm{kg} / \mathrm{d})\end{array}$ & 0.52 & 0.44 & 0.30 & 0.36 & 0.03 & 0.02 & 0.85 & 0.23 \\
\hline $\begin{array}{c}4-6 \text { week ADG } \\
(\mathrm{kg} / \mathrm{d})\end{array}$ & 0.73 & 0.68 & 0.57 & 0.52 & 0.03 & 0.02 & 0.43 & 0.99 \\
\hline $\begin{array}{c}6 \text { - } 8 \text { week ADG } \\
(\mathrm{kg} / \mathrm{d})\end{array}$ & 0.87 & 0.76 & 0.82 & 0.93 & 0.06 & 0.64 & 0.97 & 0.44 \\
\hline
\end{tabular}

Note: $\mathrm{M}$ denotes the effect of milk allowance. $\mathrm{F}$ denotes the effect of starters, and $\mathrm{M} \times \mathrm{F}$ denotes the interaction between milk allowance and starters. HMLS group: high milk (6 L) + low starch (21\%), high NDF (28\%), high molasses (10\%) starter; HMHS group: high milk (6 L) + high starch (40\%), low NDF (14\%), low molasses (5\%) starter; LMLS group: low milk (3 L) + low starch (21\%), high NDF (28\%), high molasses (10\%) starter LMHS group: low milk (3 L) + high starch (40\%), low NDF (14\%), low molasses (5\%) starter.

2 - 4 weeks of age and during 4 - 6 weeks of age $(P<0.05)$, did not affect daily weight gain during 6 - 8 weeks of age $(P>0.1)$. Different starters did not affect daily gain of calves during the trial period $(P>0.1)$.

\subsection{Stomach Tissue Development}

Table 7 shows that the low milk allowance increased the complex stomach full weight, and the reticulorumen full weight $(P<0.05)$. The low milk allowance increased the percentage of the complex stomach full weight $(P<0.05)$, increased the percentage of the reticulorumen full weight $(P<0.01)$. The low milk allowance tended to increase the percentage of reticulorumen full weight, and the volume of reticulorumen $(0.05<P<0.1)$. The milk allowance did not affect the empty body weight, the complex stomach empty weight, the percentage of the complex stomach empty weight, the reticulorumen empty weight, the omasum full weight, the percentage of the omasum full weight, the omasum empty weight, the percentage of the omasum empty weight, the abomasum full weight, the percentage of abomasum full weight over empty body weight, abomasum empty weight, the percentage of the abomasum empty weight, the abomasum volume $(P>0.1)$. The high starch low in fiber low molasses starter significantly increased the complex stomach full weight, the percentage of complex stomach full weight, the reticulorumen empty weight, the percentage of reticulorumen empty weight, the abomasum full weight, the percentage of abomasum full weight, and the reticulorumen volume $(P<0.05)$, tended to increase the complex stomach empty weight, the reticulorumen full weight, the percentage of the reticulorumen full weight, omasum full weight, the percentage of omasum full weight $(0.05<P<0.1)$. Different starters did not affect empty body weight, the percentage of complex stomach empty weight, the omasum empty weight, the percentage of the omasum empty weight, the abomasum empty weight, the percentage of the abomasum empty weight, and abomasum volume $(P>0.1)$. 
Table 7. Effect of different treatments on stomach weight of calves.

\begin{tabular}{|c|c|c|c|c|c|c|c|c|}
\hline \multirow{2}{*}{ Item } & \multicolumn{4}{|c|}{ Treatment } & \multirow{2}{*}{ SEM } & \multicolumn{3}{|c|}{$P$} \\
\hline & HMLS & HMHS & LMLS & LMHS & & M & $\mathrm{F}$ & $\mathrm{M} \times \mathrm{F}$ \\
\hline Body empty weight (kg) & 72.00 & 70.98 & 68.06 & 70.16 & 0.73 & 0.12 & 0.70 & 0.28 \\
\hline $\begin{array}{l}\text { Complex stomach full } \\
\text { weight }(\mathrm{kg})\end{array}$ & 6.87 & 8.86 & 8.65 & 10.55 & 0.49 & 0.03 & 0.02 & 0.95 \\
\hline $\begin{array}{l}\text { Complex stomach full } \\
\text { weight (\%) }\end{array}$ & 9.57 & 12.50 & 12.67 & 15.03 & 0.70 & 0.01 & 0.02 & 0.76 \\
\hline $\begin{array}{l}\text { Complex stomach } \\
\text { empty weight }(\mathrm{kg})\end{array}$ & 2.07 & 2.17 & 1.97 & 2.36 & 0.07 & 0.75 & 0.09 & 0.29 \\
\hline $\begin{array}{l}\text { Complex stomach } \\
\text { empty weight (\%) }\end{array}$ & 2.88 & 3.05 & 2.89 & 3.36 & 0.10 & 0.41 & 0.11 & 0.43 \\
\hline $\begin{array}{l}\text { Rumen-reticulum full } \\
\text { weight }(\mathrm{kg})\end{array}$ & 4.92 & 6.22 & 6.74 & 8.25 & 0.45 & 0.02 & 0.06 & 0.87 \\
\hline $\begin{array}{l}\text { Rumen-reticulum full } \\
\text { weight (\%) }\end{array}$ & 6.84 & 8.77 & 9.86 & 11.75 & 0.64 & 0.01 & 0.05 & 0.98 \\
\hline $\begin{array}{l}\text { Rumen-reticulum } \\
\text { empty weight }(\mathrm{kg})\end{array}$ & 1.21 & 1.28 & 1.22 & 1.48 & 0.04 & 0.17 & 0.04 & 0.22 \\
\hline $\begin{array}{l}\text { Rumen-reticulum } \\
\text { empty weight (\%) }\end{array}$ & 1.68 & 1.81 & 1.79 & 2.11 & 0.06 & 0.06 & 0.04 & 0.33 \\
\hline $\begin{array}{l}\text { Omasum full weight } \\
\qquad(\mathrm{kg})\end{array}$ & 0.46 & 0.65 & 0.43 & 0.57 & 0.04 & 0.51 & 0.07 & 0.76 \\
\hline Omasum full weight (\%) & 0.64 & 0.93 & 0.64 & 0.82 & 0.06 & 0.63 & 0.08 & 0.67 \\
\hline $\begin{array}{l}\text { Omasum empty weight } \\
(\mathrm{kg})\end{array}$ & 0.40 & 0.37 & 0.32 & 0.34 & 0.03 & 0.46 & 0.98 & 0.70 \\
\hline $\begin{array}{c}\text { Omasum empty weight } \\
(\%)\end{array}$ & 0.55 & 0.52 & 0.46 & 0.49 & 0.04 & 0.55 & 0.96 & 0.79 \\
\hline $\begin{array}{l}\text { Abomasum full weight } \\
\qquad(\mathrm{kg})\end{array}$ & 1.36 & 1.95 & 1.45 & 1.72 & 0.10 & 0.70 & 0.04 & 0.38 \\
\hline $\begin{array}{l}\text { Abomasum full weight } \\
(\%)\end{array}$ & 1.89 & 2.73 & 2.14 & 2.45 & 0.14 & 0.94 & 0.04 & 0.30 \\
\hline $\begin{array}{l}\text { Abomasum empty } \\
\text { weight }(\mathrm{kg})\end{array}$ & 0.47 & 0.52 & 0.43 & 0.53 & 0.02 & 0.86 & 0.17 & 0.61 \\
\hline $\begin{array}{l}\text { Abomasum empty } \\
\text { weight (\%) }\end{array}$ & 0.65 & 0.73 & 0.63 & 0.76 & 0.03 & 0.88 & 0.16 & 0.72 \\
\hline $\begin{array}{l}\text { Rumen-reticulum } \\
\text { volume (L) }\end{array}$ & 8.31 & 10.51 & 10.40 & 14.59 & 0.90 & 0.05 & 0.04 & 0.49 \\
\hline Abomasum volume (L) & 2.03 & 1.89 & 2.45 & 3.33 & 0.29 & 0.12 & 0.51 & 0.36 \\
\hline
\end{tabular}

Note: The percentage is the organ weight over the body empty weight. The empty body weight means body weight minus chyme. The organ empty weight means the weight of an organ after washed with water. The full weight means empty weight plus chyme weight. $M$ denotes the effect of milk allowance. $F$ denotes effect of starters and $\mathrm{M} \times \mathrm{F}$ denotes the interaction between milk allowance and starters. HMLS group: high milk (6 L) + low starch (21\%), high NDF (28\%), high molasses (10\%) starter; HMHS group: high milk (6 L) + high starch (40\%), low NDF (14\%), low molasses (5\%) starter; LMLS group: low milk (3 L) + low starch (21\%), high NDF (28\%), high molasses (10\%) starter LMHS group: low milk (3 L) + high starch (40\%), low NDF (14\%), low molasses (5\%) starter.

\subsection{Effect of Different Dietary Treatments on Calf Intestinal Tissue Morphology}

Table 8 shows the effect of different dietary treatments on intestinal tissue morphological characteristics. The low allowance increased the length and full weight of the small intestine, the percentage of small intestine full weight over empty body weight $(P<0.01)$. The milk allowance did not affect large intestine development $(P>0.1)$. The low starch high fiber high molasses starter increased 
Table 8. Effect of different treatments on intestinal weight of calves.

\begin{tabular}{|c|c|c|c|c|c|c|c|c|}
\hline & \multicolumn{4}{|c|}{ Treatment } & \multirow{2}{*}{ SEM } & \multicolumn{3}{|c|}{$P$} \\
\hline & HMLS & HMHS & LMLS & LMHS & & M & $\mathrm{F}$ & $\mathrm{M} \times \mathrm{F}$ \\
\hline $\begin{array}{l}\text { Small intestine length } \\
\qquad(\mathrm{cm})\end{array}$ & 1935 & 1795 & 2170 & 2060 & 47.54 & 0.00 & 0.04 & 0.77 \\
\hline $\begin{array}{l}\text { Small intestine full weight } \\
\qquad(\mathrm{kg})\end{array}$ & 3.96 & 3.75 & 4.39 & 4.18 & 0.08 & 0.00 & 0.05 & 0.97 \\
\hline $\begin{array}{l}\text { Small intestine full weight } \\
\text { (\%) }\end{array}$ & 5.51 & 5.30 & 6.45 & 5.96 & 0.15 & 0.00 & 0.05 & 0.38 \\
\hline $\begin{array}{l}\text { Small intestine empty } \\
\text { weight }(\mathrm{kg})\end{array}$ & 2.59 & 2.34 & 2.77 & 2.40 & 0.10 & 0.58 & 0.18 & 0.79 \\
\hline $\begin{array}{l}\text { Small intestine empty } \\
\text { weight (\%) }\end{array}$ & 3.60 & 3.30 & 4.06 & 3.42 & 0.15 & 0.34 & 0.14 & 0.58 \\
\hline $\begin{array}{l}\text { Large intestine length } \\
\qquad(\mathrm{cm})\end{array}$ & 392.17 & 368.23 & 342.50 & 399.33 & 14.27 & 0.76 & 0.59 & 0.20 \\
\hline $\begin{array}{l}\text { Large intestine full weight } \\
\qquad(\mathrm{kg})\end{array}$ & 1.88 & 2.21 & 1.85 & 1.97 & 0.07 & 0.34 & 0.12 & 0.43 \\
\hline $\begin{array}{l}\text { Large intestine full weight } \\
\text { (\%) }\end{array}$ & 2.61 & 3.11 & 2.73 & 2.81 & 0.10 & 0.66 & 0.17 & 0.31 \\
\hline $\begin{array}{l}\text { Large intestine empty } \\
\text { weight }(\mathrm{kg})\end{array}$ & 1.05 & 1.25 & 1.08 & 1.14 & 0.06 & 0.79 & 0.39 & 0.61 \\
\hline $\begin{array}{l}\text { Large intestine empty } \\
\text { weight (\%) }\end{array}$ & 1.45 & 1.76 & 1.59 & 1.62 & 0.09 & 0.99 & 0.41 & 0.48 \\
\hline
\end{tabular}

Note: The percentage is over body empty weight, which is body weight minus chime weight. The empty organ weight means the weight of organs washed with water. The full weight means empty weight plus chyme weight. $\mathrm{M}$ denotes the effect of milk allowance, $\mathrm{F}$ denotes the effect of type of starter and $\mathrm{M} \times \mathrm{F}$ means interaction between milk allowance and starters. HMLS group: high milk (6 L) + low starch (21\%), high NDF $(28 \%)$, high molasses (10\%) starter; HMHS group: high milk (6 L) + high starch $(40 \%)$, low NDF (14\%), low molasses (5\%) starter; LMLS group: low milk (3 L) + low starch (21\%), high NDF (28\%), high molasses (10\%) starter LMHS group: low milk (3 L) + high starch (40\%), low NDF (14\%), low molasses (5\%) starter.

the small intestine length $(P<0.05)$ and the percentage of small intestine full weight $(P=0.05)$, tended to increase the small intestine full weight $(0.05<P<$ $0.1)$. Starters had no effect on the small intestine empty weight, the percentage of small intestine empty weight, and large intestine development indicators $(P>$ $0.1)$.

\subsection{Effect of Different Dietary Treatments on VFA and pH of the Rumen Fluid}

Table 9 shows the effect of dietary treatments on concentrations of rumen fluid volatile fatty acids. The high milk allowance increased acetic acid concentration $(P<0.05)$, tended to reduce ruminal $\mathrm{pH}(0.05<P<0.01)$, but did not affect the concentrations of propionic acid, butyric acid, pentanoic acid, hexanoic acid and total VFA $(P>0.1)$. The low starch high fiber high molasses starter increased acetic acid concentration $(P<0.01)$, tended to reduce propionic acid concentration $(0.05<P<0.1)$, increased the rumen fluid $\mathrm{pH}(P<0.01)$. Starters had no effect on concentrations of butyric acid, pentanoic acid and total VFA $(P>0.1)$.

\subsection{Serum Parameters}

Table 10 shows the effect of different dietary treatments on calf serum parameters. 
Table 9. Effect of different treatments on rumen VFA and $\mathrm{pH}$ of calves.

\begin{tabular}{|c|c|c|c|c|c|c|c|c|}
\hline & \multicolumn{4}{|c|}{ Treatment } & \multirow{2}{*}{ SEM } & \multicolumn{3}{|c|}{$P$} \\
\hline & HMLS & HMHS & LMLS & LMHS & & M & $\mathrm{F}$ & $\mathrm{M} \times \mathrm{F}$ \\
\hline $\begin{array}{c}\text { Acetic acid } \\
(\mathrm{mmol} / \mathrm{L})\end{array}$ & 41.60 & 32.08 & 33.98 & 29.48 & 1.61 & 0.03 & 0.00 & 0.25 \\
\hline $\begin{array}{l}\text { Propionic acid } \\
\quad(\mathrm{mmol} / \mathrm{L})\end{array}$ & 28.39 & 33.12 & 22.49 & 31.97 & 1.84 & 0.30 & 0.06 & 0.48 \\
\hline $\begin{array}{l}\text { Butyric acid } \\
(\mathrm{mmol} / \mathrm{L})\end{array}$ & 12.34 & 10.73 & 9.52 & 12.24 & 1.05 & 0.78 & 0.82 & 0.38 \\
\hline $\begin{array}{l}\text { Pentoic acid } \\
(\mathrm{mmol} / \mathrm{L})\end{array}$ & 1.79 & 0.90 & 0.78 & 1.75 & 0.27 & 0.88 & 0.95 & 0.13 \\
\hline $\begin{array}{l}\text { Hexanoic acid } \\
\quad(\mathrm{mmol} / \mathrm{L})\end{array}$ & 3.32 & 2.84 & 2.15 & 2.72 & 0.26 & 0.25 & 0.92 & 0.35 \\
\hline $\begin{array}{l}\text { Total VFA } \\
(\mathrm{mmol} / \mathrm{L})\end{array}$ & 87.45 & 79.67 & 68.93 & 78.16 & 3.35 & 0.15 & 0.91 & 0.22 \\
\hline $\mathrm{pH}$ & 5.50 & 5.34 & 5.57 & 5.48 & 0.03 & 0.09 & 0.05 & 0.61 \\
\hline
\end{tabular}

Note: $\mathrm{M}$ denotes the effect of milk allowance. $\mathrm{F}$ denotes the effect of starters and $\mathrm{M} \times \mathrm{F}$ denotes the interaction between milk allowance and starters. HMLS group: high milk (6 L) + low starch (21\%), high NDF (28\%), high molasses (10\%) starter; HMHS group: high milk (6L) + high starch (40\%), low NDF (14\%), low molasses (5\%) starter; LMLS group: low milk (3 L) + low starch (21\%), high NDF (28\%), high molasses (10\%) starter LMHS group: low milk (3 L) + high starch (40\%), low NDF (14\%), low molasses (5\%) starter.

Table 10. Effect of different treatments on serum parameters of calves.

\begin{tabular}{|c|c|c|c|c|c|c|c|c|}
\hline & \multicolumn{4}{|c|}{ Treatment } & \multirow{2}{*}{ SEM } & \multicolumn{3}{|c|}{$P$} \\
\hline & HMLS & HMHS & LMLS & LMHS & & M & $\mathrm{F}$ & $\mathrm{M} \times \mathrm{F}$ \\
\hline $\begin{array}{l}\text { Blood glucose } \\
(\mathrm{mmol} / \mathrm{L})\end{array}$ & 4.44 & 4.47 & 3.12 & 4.06 & 0.25 & 0.09 & 0.31 & 0.34 \\
\hline Total protein $(\mathrm{g} / \mathrm{L})$ & 56.93 & 60.10 & 59.67 & 55.28 & 1.00 & 0.59 & 0.75 & 0.08 \\
\hline Urea $(\mathrm{mmol} / \mathrm{L})$ & 2.31 & 1.98 & 2.45 & 3.75 & 0.29 & 0.07 & 0.33 & 0.11 \\
\hline Insulin (mIU/L) & 26.17 & 26.13 & 29.20 & 27.12 & 0.66 & 0.16 & 0.43 & 0.45 \\
\hline IGF-1(ng/mL) & 33.82 & 56.76 & 71.05 & 66.39 & 6.95 & 0.10 & 0.49 & 0.31 \\
\hline BHBA(ug/mL) & 61.74 & 66.99 & 58.86 & 62.02 & 1.53 & 0.22 & 0.19 & 0.73 \\
\hline
\end{tabular}

Note: $\mathrm{M}$ denotes the effect of milk allowance. $\mathrm{F}$ denotes the effect of starters and $\mathrm{M} \times \mathrm{F}$ denotes the interaction between milk allowance and starters. HMLS group: high milk (6 L) + low starch (21\%), high NDF (28\%), high molasses (10\%) starter; HMHS group: high milk (6 L) + high starch (40\%), low NDF (14\%), low molasses (5\%) starter; LMLS group: low milk (3 L) + low starch (21\%), high NDF (28\%), high molasses (10\%) starter LMHS group: low milk (3 L) + high starch (40\%), low NDF (14\%), low molasses (5\%) starter.

The high allowance tended to elevate serum glucose concentrations $(0.05<P<$ $0.1)$, tended to reduce urea concentrations $(0.05<P<0.1)$, while not affecting serum total proteins, insulin, IGF-1, and BHBA contents $(P>0.1)$. Starters had no effect $(P>0.1)$ on serum indexes, however, there was a tendency of interaction between milk allowance and starters on serum total protein contents. Serum total protein contents tended to increase in HMHS and LMLS calves $(0.05<P<$ $0.1)$.

\subsection{Rumen Papillae Development}

Table 11 shows the effect of different dietary treatments on rumen papilla 
Table 11. Effect of different treatments on papilla development of calves.

\begin{tabular}{|c|c|c|c|c|c|c|c|c|c|}
\hline & & \multicolumn{4}{|c|}{ Treatment } & \multirow{2}{*}{ SEM } & \multicolumn{3}{|c|}{$P$} \\
\hline & & HMLS & HMHS & LMLS & LMHS & & M & F & $\mathrm{M} \times \mathrm{F}$ \\
\hline \multirow{3}{*}{$\begin{array}{c}\text { Anterior } \\
\text { ventral } \\
\text { sac }\end{array}$} & $\begin{array}{l}\text { Papilla length } \\
\text { (um) }\end{array}$ & 3267 & 2020 & 1743 & 1833 & 230 & 0.03 & 0.11 & 0.07 \\
\hline & $\begin{array}{l}\text { Papilla width } \\
\text { (um) }\end{array}$ & 138 & 169 & 159 & 170 & 11 & 0.68 & 0.41 & 0.69 \\
\hline & $\begin{array}{l}\text { Muscle } \\
\text { thickness } \\
\text { (um) }\end{array}$ & 1557 & 1501 & 1906 & 1378 & 113 & 0.63 & 0.23 & 0.33 \\
\hline \multirow{3}{*}{$\begin{array}{l}\text { Posterior } \\
\text { ventral } \\
\text { sac }\end{array}$} & $\begin{array}{l}\text { Papilla length } \\
\text { (um) }\end{array}$ & 1473 & 1813 & 2733 & 2427 & 225 & 0.05 & 0.97 & 0.44 \\
\hline & $\begin{array}{l}\text { Papilla width } \\
\quad \text { (um) }\end{array}$ & 227 & 183 & 197 & 181 & 11 & 0.51 & 0.22 & 0.55 \\
\hline & $\begin{array}{l}\text { Muscle } \\
\text { thickness } \\
\text { (um) }\end{array}$ & 1631 & 1185 & 1259 & 1181 & 112 & 0.43 & 0.28 & 0.44 \\
\hline \multirow{3}{*}{$\begin{array}{l}\text { Anterior } \\
\text { dorsal sac }\end{array}$} & $\begin{array}{l}\text { Papilla length } \\
\text { (um) }\end{array}$ & 3940 & 2590 & 3703 & 4520 & 234 & 0.01 & 0.30 & 0.00 \\
\hline & $\begin{array}{l}\text { Papilla width } \\
\text { (um) }\end{array}$ & 175 & 278 & 241 & 194 & 23 & 0.84 & 0.56 & 0.14 \\
\hline & $\begin{array}{l}\text { Muscle } \\
\text { thickness } \\
\text { (um) }\end{array}$ & 1551 & 1717 & 1918 & 1417 & 91 & 0.84 & 0.35 & 0.08 \\
\hline \multirow{3}{*}{$\begin{array}{l}\text { Posterior } \\
\text { dorsal sac }\end{array}$} & $\begin{array}{l}\text { Papilla length } \\
\text { (um) }\end{array}$ & 2543 & 1907 & 2190 & 2480 & 181 & 0.78 & 0.66 & 0.26 \\
\hline & $\begin{array}{l}\text { Papilla width } \\
\text { (um) }\end{array}$ & 187 & 290 & 198 & 228 & 14 & 0.12 & 0.00 & 0.04 \\
\hline & $\begin{array}{l}\text { Muscle } \\
\text { thickness } \\
\text { (um) }\end{array}$ & 753 & 1220 & 1812 & 1113 & 139 & 0.03 & 0.54 & 0.01 \\
\hline
\end{tabular}

Notes: $\mathrm{M}$ denotes the effect of milk allowance, $\mathrm{F}$ denotes the effect of starters and $\mathrm{M} \times \mathrm{F}$ denotes the interaction between milk allowance and starters. HMLS group: high milk (6 L) + low starch (21\%), high NDF (28\%), high molasses (10\%) starter; HMHS group: high milk (6L) + high starch (40\%), low NDF (14\%), low molasses (5\%) starter; LMLS group: low milk (3 L) + low starch (21\%), high NDF (28\%), high molasses (10\%) starter LMHS group: low milk (3 L) + high starch (40\%), low NDF (14\%), low molasses (5\%) starter.

length, width and muscle thickness. The high milk allowance increased papilla lengths in the anterior ventral sac $(P<0.05)$, but reduced papilla lengths in the posterior ventral sac $(P<0.05)$. The milk allowance did not affect the papilla width and muscle thickness in the anterior ventral sac, and the papilla width and muscle thickness of the posterior ventral sac $(P>0.1)$. Starters did not affect papilla width, muscle thickness in anterior sacs and the posterior dorsal sac, nor affected papilla length of posterior dorsal sac $(P>0.1)$. There were interactions between milk allowance and starters for the papilla length in the anterior dorsal sac, and the papilla width and muscle thickness of the posterior dorsal sac $(P<$ 0.05). There were no significant differences in muscle thickness in the posterior blind sac. With high starch low in fiber low molasses starters, the milk allowance 
did not affect the papilla length of the anterior dorsal sac, but the low milk allowance increased the muscle thickness of the dorsal sac $(P<0.05)$. When fed 6 L milk, the low starch high fiber high molasses starter reduced the papilla length of the anterior dorsal sac $(P<0.05)$, reduced the papilla width in the posterior dorsal sac $(P<0.05)$, however, under $3 \mathrm{~L}$ milk feeding conditions, the low starch high fiber high molasses starter reduced papilla length in the dorsal sac, increased the muscle thickness in the posterior dorsal sac $(P<0.05)$, but did not affect the width of papillae in the posterior dorsal sac (Figure 1).

\subsection{Intestinal Villus Length, Width, Muscle Thickness}

Table 12 shows the effect of different dietary treatments on calf intestinal villi

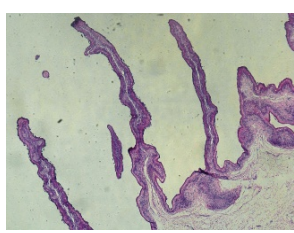

HMLS

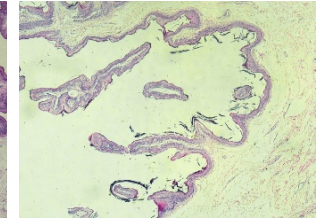

HMHS

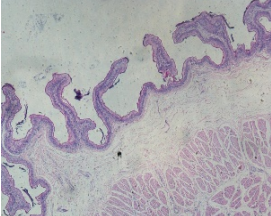

LMLS

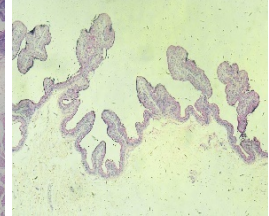

LMHS

Figure 1. Examples of papillae in the ventral sac of rumen $(\times 100)$. HMLS group: high milk (6 L) + low starch (21\%), high NDF (28\%), high molasses (10\%) starter; HMHS group: high milk (6 L) + high starch (40\%), low NDF (14\%), low molasses (5\%) starter; LMLS group: low milk (3 L) + low starch (21\%), high NDF (28\%), high molasses (10\%) starter LMHS group: low milk (3 L) + high starch (40\%), low NDF (14\%), low molasses (5\%) starter.

Table 12. Effect of different treatments on intestinal villus development in calves.

\begin{tabular}{|c|c|c|c|c|c|c|c|c|}
\hline & \multicolumn{4}{|c|}{ Treatment } & \multirow{2}{*}{ SEM } & \multicolumn{3}{|c|}{$P$} \\
\hline & HMLS & HMHS & LMLS & LMHS & & M & $\mathrm{F}$ & $\mathrm{M} \times \mathrm{F}$ \\
\hline $\begin{array}{l}\text { Duodenal villus length } \\
\text { (um) }\end{array}$ & 505.92 & 477.94 & 513.98 & 422.10 & 20.06 & 0.56 & 0.17 & 0.44 \\
\hline $\begin{array}{l}\text { Duodenal villus width } \\
\text { (um) }\end{array}$ & 86.46 & 95.17 & 84.93 & 78.09 & 3.59 & 0.23 & 0.90 & 0.31 \\
\hline $\begin{array}{l}\text { Duodenal muscle } \\
\text { thickness (um) }\end{array}$ & 411.62 & 349.90 & 546.77 & 460.02 & 38.08 & 0.13 & 0.34 & 0.87 \\
\hline $\begin{array}{l}\text { Jejunum villus length } \\
\text { (um) }\end{array}$ & 418.81 & 389.25 & 443.39 & 461.86 & 19.57 & 0.28 & 0.90 & 0.58 \\
\hline $\begin{array}{l}\text { Jejunum villus width } \\
\text { (um) }\end{array}$ & 95.06 & 105.70 & 86.83 & 86.09 & 3.04 & 0.01 & 0.30 & 0.23 \\
\hline $\begin{array}{l}\text { Jejunum muscle } \\
\text { thickness (um) }\end{array}$ & 222.45 & 281.65 & 366.74 & 366.28 & 32.49 & 0.11 & 0.65 & 0.65 \\
\hline Ileal villus length (um) & 456.40 & 525.44 & 414.87 & 425.83 & 20.14 & 0.09 & 0.30 & 0.44 \\
\hline Ileal villus width (um) & 92.68 & 87.08 & 95.91 & 84.65 & 2.40 & 0.93 & 0.11 & 0.56 \\
\hline $\begin{array}{l}\text { Ileal muscle thickness } \\
\text { (um) }\end{array}$ & 458.88 & 402.63 & 381.86 & 456.87 & 28.08 & 0.86 & 0.88 & 0.32 \\
\hline
\end{tabular}

Notes: $\mathrm{M}$ denotes the effect of milk allowance, $\mathrm{F}$ denotes the effect of starters and $\mathrm{M} \times \mathrm{F}$ denotes the interaction between milk allowance and starters. HMLS group: high milk $(6 \mathrm{~L})+$ low starch $(21 \%)$, high NDF (28\%), high molasses (10\%) starter; HMHS group: high milk (6L) + high starch (40\%), low NDF (14\%), low molasses (5\%) starter; LMLS group: low milk (3 L) + low starch (21\%), high NDF (28\%), high molasses (10\%) starter LMHS group: low milk (3 L) + high starch (40\%), low NDF (14\%), low molasses (5\%) starter. 
segment length, width and muscle thickness. The high milk allowance increased villus width in the jejunum $(P<0.05)$, tended to increase the villi length in the ilium $(0.05<P<0.1)$, but milk allowance did not affect the duodenal villus length, the width, the muscle thickness, the jejunum villus length, the muscle thickness and width and the muscle thickness of the ilium $(P>0.1)$. Starters had no effects $(P>0.1)$ the villus length, width and muscle thickness in the small intestine (Figure 2).

\section{Discussion}

\subsection{Effect of Dietary Treatments on Feed Intake}

Calves are capable of taking a certain amount of solid feed from 14 days of age [7] [8]. When the amount milk is reduced or during weaning, calves rapidly increase solid feed intake [9] [10] [11]. In this experiment, milk allowance affected concentrate DMI and total DMI. In the process of growth and development, calves meet the energy requirement mainly from milk and solid feed. When the amount of milk fed is reduced, calves must consume more solid feed to compensate for the energy deficit. So low milk allowance increased concentrate DMI and total DMI. This is consistent with previous findings [3]. Traditional high-starch starters and low starch high fiber high molasses starters had no effect on the concentrate DMI and total DMI. Studies have shown that a certain amount of fiber in starters can increase the DMI of calves [12] [13]. Lesmeister

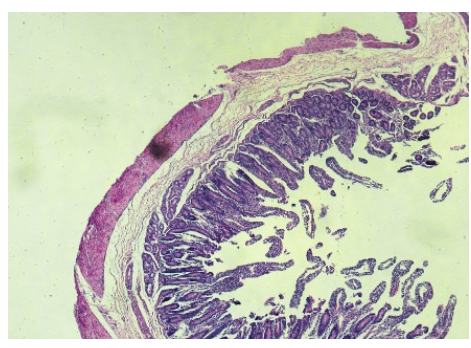

HMLS

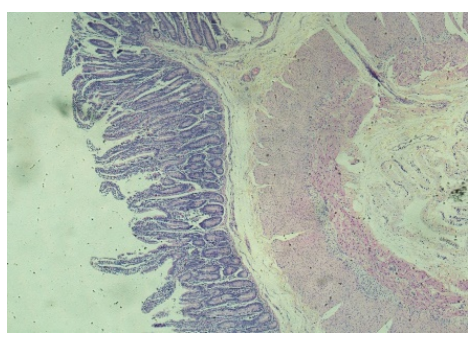

LMLS

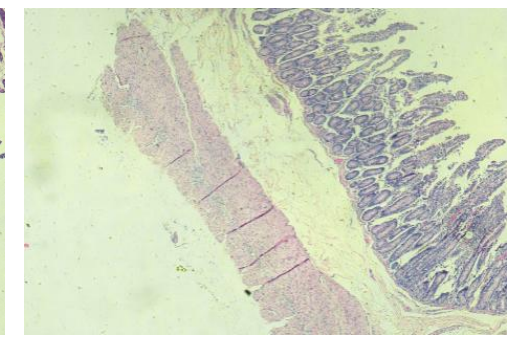

HMHS

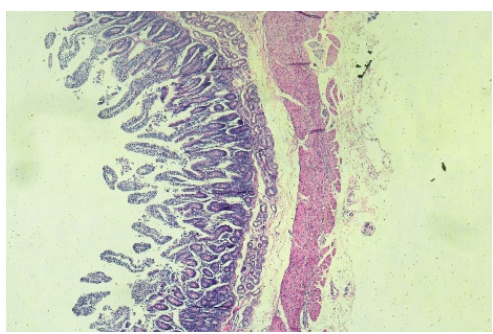

LMHS

Figure 2. Examples of tissue sections showing duodenal villi $(\times 100)$. HMLS group: high milk (6 L) + low starch (21\%), high NDF (28\%), high molasses (10\%) starter; HMHS group: high milk (6 L) + high starch (40\%), low NDF (14\%), low molasses (5\%) starter; LMLS group: low milk (3 L ) + low starch (21\%), high NDF (28\%), high molasses (10\%) starter LMHS group: low milk (3 L) + high starch (40\%), low NDF (14\%), low molasses (5\%) starter. 
showed that a high amount of molasses (12\% molasses) in starters reduced DMI [4]. In our trial, high fiber high molasses starters may offset feed intake in opposite ways, resulting in no changes on DMI of calves.

\subsection{Influence Different Dietary Treatments on Body Sizes}

Calves body sizes (body height, body length, girth, cannon circumference) increase along with increasing age. Although body sizes are mainly affected by genetic potential, nutrition and feeding management can promote or hinder the genetic potential to be realized [14]. During early calf growth, the foraging mode of calves is influenced by increasing age, and calves of different dietary treatments exhibited different influences on body sizes during each age period. In this study, the high milk allowance increased significantly the body height at 4-week-old, increased the heart girth at 6 weeks of age, increased cannon circumference at 4, 6, 8 weeks of age. This is because calves mainly consume milk in the early development. In this case, the milk allowance had a larger impact on body sizes of calves. The higher milk allowance satisfied calf nutritional and energy needs, thus calves grew faster. When calves were 6 to 8 weeks of age, their reticulorumen had developed much better than before, and rumen microbial flora was also more robust. Their ability to ferment foodstuff became stronger. Thus, the low milk group consumed greater amounts of starter. Starters supplied energy and nutrient to make up for deficiencies due to low milk intake. At the same time, the milk allowance did not affect calf body height and girth at 8 weeks of age, indicating that the milk allowance mainly affects body height ( 2 - 4 weeks old) and girth (4 - 6 weeks old). Starters mainly affect body height and heart girth at 6 to 8 weeks of age. Our study shows that different types of calf starters have no significant effect on growth performance (body height, body length, girth, cannon circumference). Because different starters had no significant effect, both starters are able to meet the needs of the energy for growth and nutrients.

\subsection{Effect of Different Dietary Treatments on Daily Gain}

Early calves have less well developed rumen, rumen microbial flora, cannot properly digest starter. At this stage, the energy and nutrients needs of calves are mainly from milk. Therefore, milk allowance in early stage has the greatest impact on calf weight. Our study shows that the high milk allowance significantly increased ADG during 2 to 4,4 to 6 weeks of development. Many studies in recent years also showed that an increase in milk allowance will increase the body weight of calves [15] [16]. In our trial, milk allowance had no significant effect on the ADG of calves during 6 to 8 weeks of age. When calves become older, their rumen is better developed, and their rumen microflora becomes more complete. After 6 weeks of age, calves consume much more starter, and their rumen microorganisms can well degrade the starter ingested. At this stage, the energy and nutrients are supplied not only from milk, but also from the starter 
diet. Thus, at this stage, starters also play an important role in promoting calf daily gain. Suárez showed that, compared with their high starch $(592.8 \mathrm{~g} / \mathrm{kg}$ of DM) low fiber ( $94 \mathrm{~g} / \mathrm{kg}$ of DM) starters, their low starch (107.9 g/kg of DM) high fiber (493 $\mathrm{g} / \mathrm{kg}$ of DM) starters increased weight gain [17]. Lesmeister showed that, a high amount of molasses ( $12 \%$ molasses) in a starter tended to decrease weight gain [4]. In our experiment, different starters had no significant effect on the daily gain of calves at all stages. Our low starch high fiber high molasses starters not only increased the fiber content, but also increased the molasses content. Probably due to the higher fiber and higher molasses cancel out the effects of each other, without affecting weight gain.

\subsection{Effect of Different Dietary Treatments on Calf Stomach Tissue Development}

In our trial, the low milk allowance increased the complex stomach full weight and the reticulorumen weight, increased the percentage of the complex stomach full weight, but did not affect the reticulorumen empty weight and the complex stomach empty weight. The abomasum full weight, the omasum full weight, the abomasum empty weight and the omasum empty weight were unaffected by the milk allowance. This shows that the low milk allowance increased the intake of solid feed, thereby increased the time by which chyme stays in the stomach. Increases in the reticulorumen full weight and the complex stomach full weight were merely caused by the increase in the amount of chyme. Likely, due to a lower chyme content in the omasum, there were no significant influence of milk allowance on the omasum full weight. Kristensen also showed that the reticulorumen full weight and the complex stomach full weight increased when the milk allowance was reduced, while the abomasum full weight was not affected by milk allowance [3]. Due to increased solid feed intake, lower milk allowance increased the extent of reticulorumen fullness, resulting in the tendency of increase in the reticulorumen volume. At the same time, a low milk allowance tends to increase the percentage of reticulorumen empty weight over body weight. This suggests that low milk allowance tends to promote reticulorumen development. In our trial, low starch high fiber high molasses starter diets significantly reduced the abomasum full weight and the complex stomach full weight, but had no significant effect on empty weights of the complex stomach and abomasum. This indicates that different starters mainly affects the chyme content of the stomach and abomasum, have no effect on the structural development of the abomasum and the complex stomach. At the same time, low starch high fiber high molasses starter diets had a tendency to lower reticulorumen full weight, reduced reticulorumen empty weight and the percentage of complex stomach empty weight over empty body weight, reduced reticulorumen volume. Studies have shown that, when starters contain less than $5 \%-6 \%$ of crude fiber content, it will not bring good benefits to calf production, and more will likely make rumen bulging [18]. In our experiment, the traditional high starch starters contained less fiber, calves may be bulging prone which may cause reticulorumen volume increase. 


\subsection{Effect of Different Dietary Treatments on Calf Intestinal Tissue}

Newborn calves are similar to monogastric animals in digestive function. At this stage, calf intestinal weight comprises a large proportion of the entire digestive tract. Along with the increase of solid feed intake, the proportion of reticulorumen weight gradually increases, and the proportion of the small intestine weight become smaller, while the proportion of the large intestine weight keep unchanged [19]. In our trial, the low milk allowance significantly increased the length of the small intestine, which may be related to the feed intake. The low milk allowance significantly increased the small intestine full weight and small intestine full weight percentage, but had no significant effect on the small intestine empty weight. This is because the low milk allowance increased the solid feed intake [9] [11], which led to an increase in chyme retention time in the small intestine, thus increased the full weight of the small intestine. For any animal, when the quality of the diet declines, in order to meet their energy needs for growth, it is necessary to adjust its own mechanisms to adapt to the diet, such as increasing the retention time of nutrients, increasing nutrient absorption area and the like. In our trial, the low-starch high fiber high molasses starter diets increased the length and the full weight of the small intestine. This is related to the high fiber content of diet. Many studies have shown that, the increase of dietary fiber content is accompanied by increases in length, weight, volume of the digestive tract. Wang showed that supplementation with alfalfa hay significantly increased the length of the small intestine (20.8 vs. $17.1 \mathrm{~m}, P<0.01)$ [20], and that the length of the small intestine significantly increased along with age increase. Pond showed that high alfalfa diets increased the relative weight of the stomach and small intestine in adult pigs [21]. In our trial, the low starch high fiber high molasses starter had no significant effect on the small intestine empty weight.

\subsection{Influence of Different Dietary Treatments on Rumen Fluid pH and VFA}

In our trial, the milk allowance had no significant effect on the amount of propionic acid, butyric acid, pentanoic acid and total VFA, which is consistent with the study by Kristensen [3]. However, the low milk allowance reduced the concentration of acetic acid, indicating that the added amount of milk does not affect the concentration of various VFA other than acetic acid via solid feed intake. Low starch high fiber high molasses starters significantly increased the concentration of acetic acid, tended to reduce the propionic acid concentration. Fiber in starter rations favors the production of acetic acid, and starch is conducive to propionic acid production, which are consistent with previous studies [22]. The rumen $\mathrm{pH}$ can be as low as 5.5 in calves fed a traditional high-starch starter, lower than the subacute rumen acidosis threshold. Although the low starch high fiber high molasses starters had no significant effect on the total VFA concentra- 
tion, they increased rumen $\mathrm{pH}$ (5.54 vs. 5.41). The rumen $\mathrm{pH}$ level is mainly affected by the proportion of a variety of VFA in rumen fluid, with the largest impact from propionate concentrations. Rumen $\mathrm{pH}$ may be negatively correlated with propionic acid concentrations [3]. Jahn showed that rumen fluid $\mathrm{pH}$ and the acetic acid concentration increase along with the fiber content [23], and the propionic acid concentration decreases along with increasing fiber contents. Therefore, the use of low starch high fiber high molasses starter to substitute traditional high-starch low-fiber low molasses starter diet can increase rumen $\mathrm{pH}$, to some extent alleviate the subacute rumen acidosis.

\subsection{Influence of Different Dietary Treatments on Serum Parameters}

In our trial, the milk allowance and starter diets did not significantly affect serum parameters, but the high milk allowance tended to increase the blood glucose concentration, which is consistent with findings of Quigley [24]. Milk ingested by newborn calves can directly enter the abomasum and small intestine through the esophageal groove. With the increase of age, calves no longer suck milk, esophageal groove closure become incomplete, and calf starter intake needs to go through the rumen microbial fermentation to produce volatile fatty acids, among which propionic acid is used by gluconeogenesis pathway to generate glucose, which enters into the blood stream as the main source of glucose. Under high milk allowance, when a calf does not ingest milk, although there is no esophageal groove reflex action, the milk flowing into the reticulorumen largely enters into the abomasum and the small intestine due to high liquidity. Lactose in milk is absorbed rapidly in the abomasum and small intestine, resulting in elevated blood sugar. Calves of high milk allowance have a tendency of reduced urea concentration, which is inconsistent with Quigley [24]. This may be related to solid feed nutrients. Starter diets did not significantly affect serum parameters, suggesting that the traditional high starch starters and low starch high fiber high molasses starters had no significant impact on animal metabolism.

\subsection{Effect of Different Dietary Treatments on Rumen Papilla Development}

Calf ruminal epithelium development is mainly affected by the concentration of ruminal VFA, especially butyrate, followed by propionic acid. Studies have shown that rumen gavage of sodium propionate, sodium butyrate can well promote the development of the rumen epithelium [25] [26]. Studies have also shown that rumen gavage of milk can increase SVFA production, and stimulate rumen papilla growth [26]. In our experiment, the rumen development was not even. The low milk allowance significantly reduced the papilla length of the anterior ventral sac, but significantly increased posterior ventral papilla length. This is inconsistent with results of studies by Kristensen and Roth [3] [6]. Probably because each part of the rumen has a different $\mathrm{pH}$, and rumen microflora is 
different, the composition of VFA was not the same. In studies by Kristensen and Roth, although their low milk allowance promoted the intake of feed solids, it did not affect the length of papillae in all parts of the rumen. Starters had no significant effect on the papilla development of anterior and posterior sacs. This indicates that a low starch high fiber high molasses starter diet could be used as a substitute for traditional high starch starters, and will not affect follicle development. Different milk allowance and different types of starters have interactions in determining the papilla length in the anterior dorsal sac, the width of the posterior rumen sac, and the posterior rumen sac muscle thickness. From a numerical point of view, the LMHS group anterior dorsal sac had the longest papilla length. This shows that under low milk allowance conditions, the traditional high starch diets achieve the best results in promoting development of dorsal sacs. In the HMHS group, papillae of the posterior dorsal sac were the highest width, and in the LMLS group, the posterior dorsal sac had the maximum muscle thickness. This shows that traditional high-starch starters are better at stimulating the papilla width under a high milk allowance, and under conditions of a low milk allowance, low starch high fiber high molasses starters are better at stimulating muscle thickness in the posterior dorsal sac.

\subsection{Effect of Different Dietary Treatments on Calf Intestinal Villi}

The small intestine is the main organ for digestion and absorption of nutrients in calves. Its villi increase the surface area of the small intestine in contacting with nutrients. Villus development is an important indicator of small intestine development. In our experiment, the high milk allowance increased the jejunum villus width tend to increase the villus length in the ileum. The villus length of the small intestine may be related to the dietary protein source. A high milk allowance increased milk protein content in the diet. Lalles and other attempted to use skim milk, hydrolyzed soy proteins, and heat treated soy flour to formulate a milk replacer, and found that calves fed skim milk had the longest villi [27]. In our trial, low starch high fiber high molasses starters had no significant effect on the development of intestinal villi, indicating that our starters have the same effect on the development of the small intestine.

\section{Conclusion}

Low starch high fiber high molasses starters, as a substitute of traditional high-starch starters, increased rumen $\mathrm{pH}$, which could prevent subacute ruminal acidosis to a certain extent, and did not affect the growth performance of calves and ruminal ventral sac papila development, but it reduced the weight of the reticulorumen and increased the length of the intestine in calves, promoting the development of the small intestine to a certain extent. The low milk allowance increased DMI, tended to promote the development of the reticulorumen, promoted papilla development in the ruminal posterior ventral sac, but hindered the development of papillae in the anterior ventral sac. The low milk allowance 
increased the length of the small intestine, but reduced the jejunum villus width.

\section{Acknowledgements}

The study was financially supported by the earmarked fund for Modern Agro-industry Technology Research System of China (CARS-36), Cattle Agro-industry Technology Research System of Shandong Province (SDAIT-12-011-06), natural science fund of China (31572427) (31372340), national Key R\&D program of China and SYL2017YSTD08. The author gratefully thanks the staff of Aote Dairy Farm, Qingdao, China.

\section{References}

[1] Warner, R.G., Flatt, W.P. and Loosli, J.K. (1956) Ruminant Nutrition, Dietary Factors Influencing Development of Ruminant Stomach. Journal of Agricultural and Food Chemistry, 4, 788-792. https://doi.org/10.1021/jf60067a003

[2] Beharka, A.A., Nagaraja, T.G., Morrill, J.L., Kennedy, G.A. and Klemm, R.D. (1998) Effects of Form of the Diet on Anatomical, Microbial, and Fermentative Development of the Rumen of Neonatal Calves. Journal of Dairy Science, 81, 1946-1955. https://doi.org/10.3168/jds.S0022-0302(98)75768-6

[3] Kristensen, N.B., Sehested, J., Jensen, S.K. and Vestergaard, M. (2007) Effect of Milk Allowance on Concentrate Intake, Ruminal Environment, and Ruminal Development in Milk-Fed Holstein Calves. Journal of Dairy Science, 90, 4346-4355. https://doi.org/10.3168/jds.2006-885

[4] Lesmeister, K.E. and Heinrichs, A.J. (2005) Effects of Adding Extra Molasses to a Texturized Calf Starter on Rumen Development, Growth Characteristics, and Blood Parameters in Neonatal Dairy Calves. Journal of Dairy Science, 88, 411-418. https://doi.org/10.3168/jds.S0022-0302(05)72702-8

[5] Baldwin, R.L., McLeod, K.R., Klotz, J.L. and Heitmann, R.N. (2004) Rumen Development, Intestinal Growth and Hepatic Metabolism in the Pre- and Postweaning Ruminant. Journal of Dairy Science, 87, E55-E65. https://doi.org/10.3168/jds.S0022-0302(04)70061-2

[6] Roth, B.A., Keil, N.M., Gygax, L. and Hillmann, E. (2009) Influence of Weaning Method on Health Status and Rumen Development in Dairy Calves. Journal of Dairy Science, 92, 645-656. https://doi.org/10.3168/jds.2008-1153

[7] Williams, P.E.V. and Frost, A.I. (1992) Feeding the Young Ruminant. Occasional Publication-British Society of Animal Production (United Kingdom), 15, 109-118.

[8] Khan, M.A., Lee, H.J., Lee, W.S., et al. (2008) Starch Source Evaluation in Calf Starter: II. Ruminal Parameters, Rumen Development, Nutrient Digestibilities, and Nitrogen Utilization in Holstein Calves. Journal of Dairy Science, 91, 1140-1149. https://doi.org/10.3168/jds.2007-0337

[9] Khan, M.A., Lee, H.J., Lee, W.S., et al. (2007) Structural Growth, Rumen Development, and Metabolic and Immune Responses of Holstein Male Calves Fed Milk through Step-Down and Conventional Methods. Journal of Dairy Science, 90, 3376-3387. https://doi.org/10.3168/jds.2007-0104

[10] Khan, M.A., Lee, H.J., Lee, W.S., et al. (2007) Pre- and Postweaning Performance of Holstein Female Calves Fed Milk through Step-Down and Conventional Methods. Journal of Dairy Science, 90, 876-885. https://doi.org/10.3168/jds.S0022-0302(07)71571-0 
[11] Jasper, J. and Weary, D.M. (2002) Effects of Ad Libitum Milk Intake on Dairy Calves. Journal of Dairy Science, 85, 3054-3058. https://doi.org/10.3168/jds.S0022-0302(02)74391-9

[12] Leibholz, J. (1975) Ground Roughage in the Diet of the Early-Weaned Calf. Animal Science, 20, 93-100. https://doi.org/10.1017/S0003356100035042

[13] Khan, M.A., Weary, D.M. and Von Keyserlingk, M.A.G. (2011) Hay Intake Improves Performance and Rumen Development of Calves Fed Higher Quantities of Milk. Journal of Dairy Science, 94, 3547-3553.

https://doi.org/10.3168/jds.2010-3871

[14] Owens, F.N., Dubeski, P. and Hanson, C.F. (1993) Factors that Alter the Growth and Development of Ruminants. Journal of Animal Science, 71, 3138-3150. https://doi.org/10.2527/1993.71113138x

[15] Bartlett, K.S., McKeith, F.K., VandeHaar, M.J., Dahl, G.E. and Drackley, J.K. (2006) Growth and Body Composition of Dairy Calves Fed Milk Replacers Containing Different Amounts of Protein at Two Feeding Rates. Journal of Animal Science, 84, 1454-1467. https://doi.org/10.2527/2006.8461454x

[16] Diaz, M.C., Van Amburgh, M.E., Smith, J.M., Kelsey, J.M. and Hutten, E.L. (2001) Composition of Growth of Holstein Calves Fed Milk Replacer from Birth to 105-Kilogram Body Weight. Journal of Dairy Science, 84, 830-842. https://doi.org/10.3168/jds.S0022-0302(01)74541-9

[17] Suárez, B.J., Van Reenen, C.G., Beldman, G., van Delen, J., Dijkstra, J. and Gerrits, W.J.J. (2006) Effects of Supplementing Concentrates Differing in Carbohydrate Composition in Veal Calf Diets: I. Animal Performance and Rumen Fermentation Characteristics. Journal of Dairy Science, 89, 4365-4375. https://doi.org/10.3168/jds.S0022-0302(06)72483-3

[18] Miller, W.J., Martin, Y.G. and Fowler, P.R. (1969) Effects of Addition of Fiber to Simplified and to Complex Starters Fed to Young Dairy Calves. Journal of Dairy Science, 52, 672-676. https://doi.org/10.3168/jds.S0022-0302(69)86626-9

[19] Liu, M.X. (1991) Ruminant Digestive Physiology. Beijing Agriculture University Press, Beijing.

[20] Wang. L.B. (2013) Feeding of Alfalfa Influence on Development of Gastrointestinal Tract of Calves on the Basis of Feeding the Starter. Ph.D. Thesis, China Agricultural University, Beijing.

[21] Pond, W.G., Jung H.G. and Varel, V.H. (1988) Effect of Dietary Fiber on Young Adult Genetically Lean, Obese and Contemporary Pigs: Body Weight, Carcass Measurements, Organ Weights and Digesta Content. Journal of Animal Science, 66, 699-706. https://doi.org/10.2527/jas1988.663699x

[22] Suárez, B.J., Van Reenen, C.G., Stockhofe, N., Dijkstra, J. and Gerrits, W.J.J. (2007) Effect of Roughage Source and Roughage to Concentrate Ratio on Animal Performance and Rumen Development in Veal Calves. Journal of Dairy Science, 90, 2390-2403. https://doi.org/10.3168/jds.2006-524

[23] Jahn, E., Chandler, P.T. and Polan, C.E. (1970) Effects of Fiber and Ratio of Starch to Sugar on Performance of Ruminating Calves. Journal of Dairy Science, 53, 466-474. https://doi.org/10.3168/jds.S0022-0302(70)86232-4

[24] Quigley, J.D., Wolfe, T.A. and Elsasser, T.H. (2006) Effects of Additional Milk Replacer Feeding on Calf Health, Growth, and Selected Blood Metabolites in Calves. Journal of Dairy Science, 89, 207-216. https://doi.org/10.3168/jds.S0022-0302(06)72085-9 
[25] Sander, E.G., Warner, R.G., Harrison, H.N. and Loosli, J.K. (1959) The Stimulatory Effect of Sodium Butyrate and Sodium Propionate on the Development of Rumen Mucosa in the Young Calf. Journal of Dairy Science, 42, 1600-1605. https://doi.org/10.3168/jds.S0022-0302(59)90772-6

[26] Tamate, H., McGilliard, A.D., Jacobson, N.L. and Getty, R. (1962) Effect of Various Dietaries on the Anatomical Development of the Stomach in the Calf. Journal of Dairy Science, 45, 408-420. https://doi.org/10.3168/jds.S0022-0302(62)89406-5

[27] Lalles, J.P., Toullec, R. and Pardal, P.B. (1995) Hydrolyzed Soy Protein Isolate Sustains High Nutritional Performance in Veal Calves. Journal of Dairy Science, 78, 194-204. https://doi.org/10.3168/jds.S0022-0302(95)76629-2 